Supporting Information

\title{
Effect of Defective Microstructure and Film Thickness on the Reflective Structural Color of Self-Assembled Colloidal Crystals
}

Tianyu Liu, ${ }^{\dagger}$ Bryan VanSaders ${ }^{\ddagger}$, Sharon C. Glotzer, ${ }^{\dagger,}, \S$ and Michael J. Solomon, $*, \dagger, \S$

${ }^{\dagger}$ Macromolecular Science and Engineering, ${ }^{\ddagger}$ Materials Science and Engineering, and *Department of Chemical Engineering, University of Michigan, Ann Arbor, Michigan 48109, United States

*email: mjsolo@umich.edu

KEYWORDS: colloidal self-assembly, evaporation, crystal film, defect, structural color, molecular dynamics, FDTD 

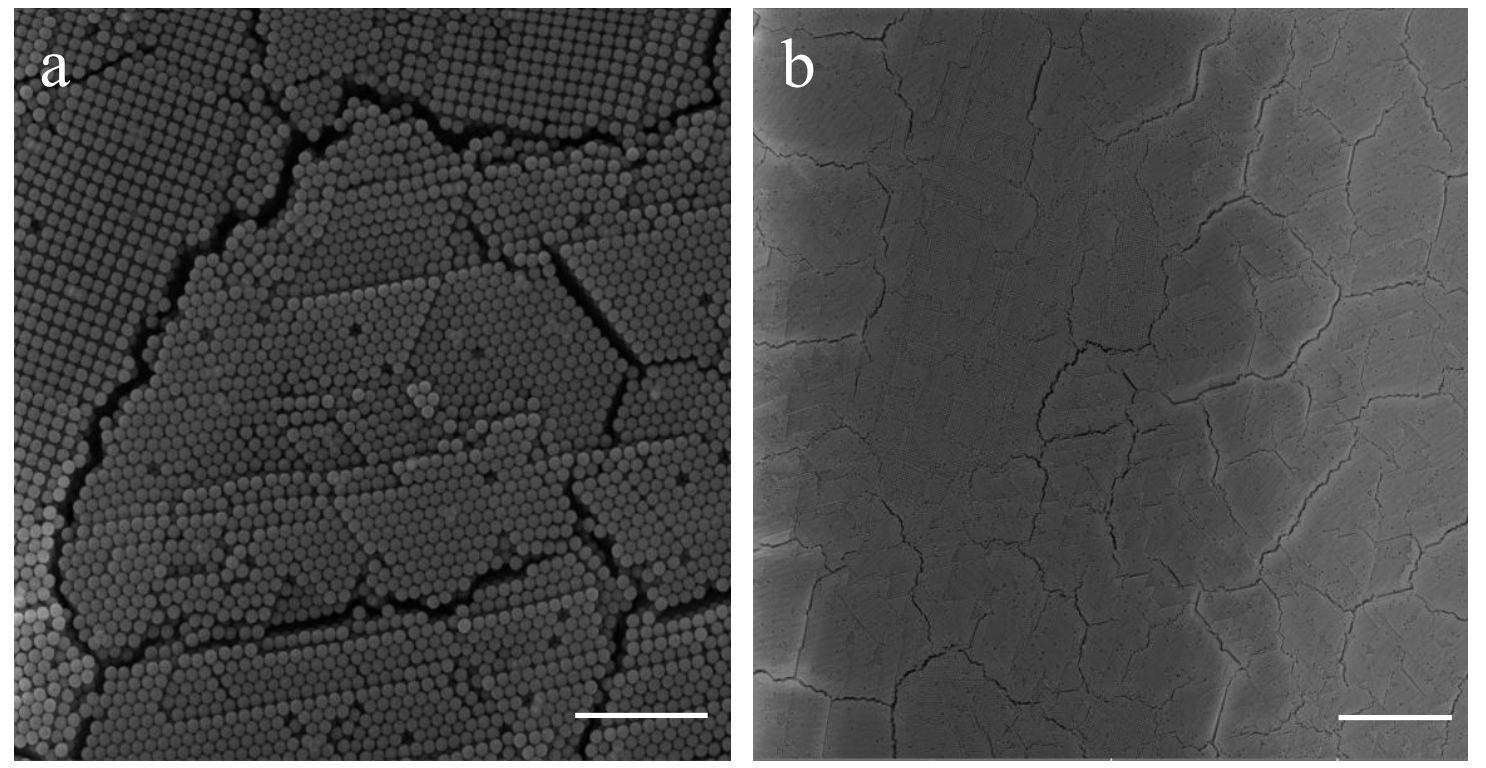

Figure S1. SEM images of the self-assembled colloidal crystals show microcracks of width less than $1 \mu \mathrm{m}$. Scale bar: (a) $2 \mu \mathrm{m}$ and (b) $10 \mu \mathrm{m}$. 

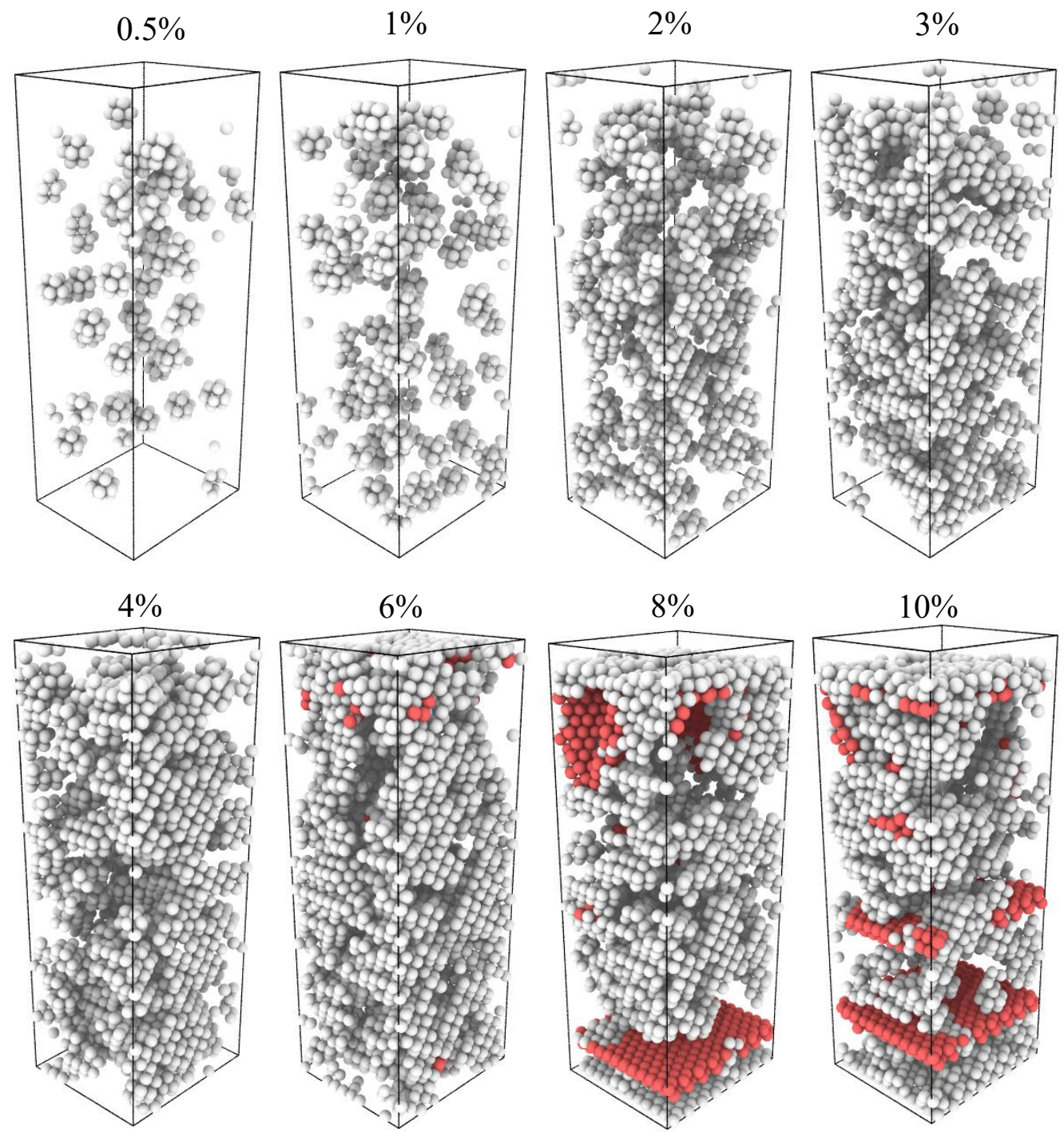

Figure S2. Simulated structures with increasing number fraction of clustered vacancies. Particles are colored by local environment ( $\mathrm{red}=\mathrm{HCP}$ and white=disordered). Colloids formed $\mathrm{FCC}$ crystals are not shown for ease of visualization. 

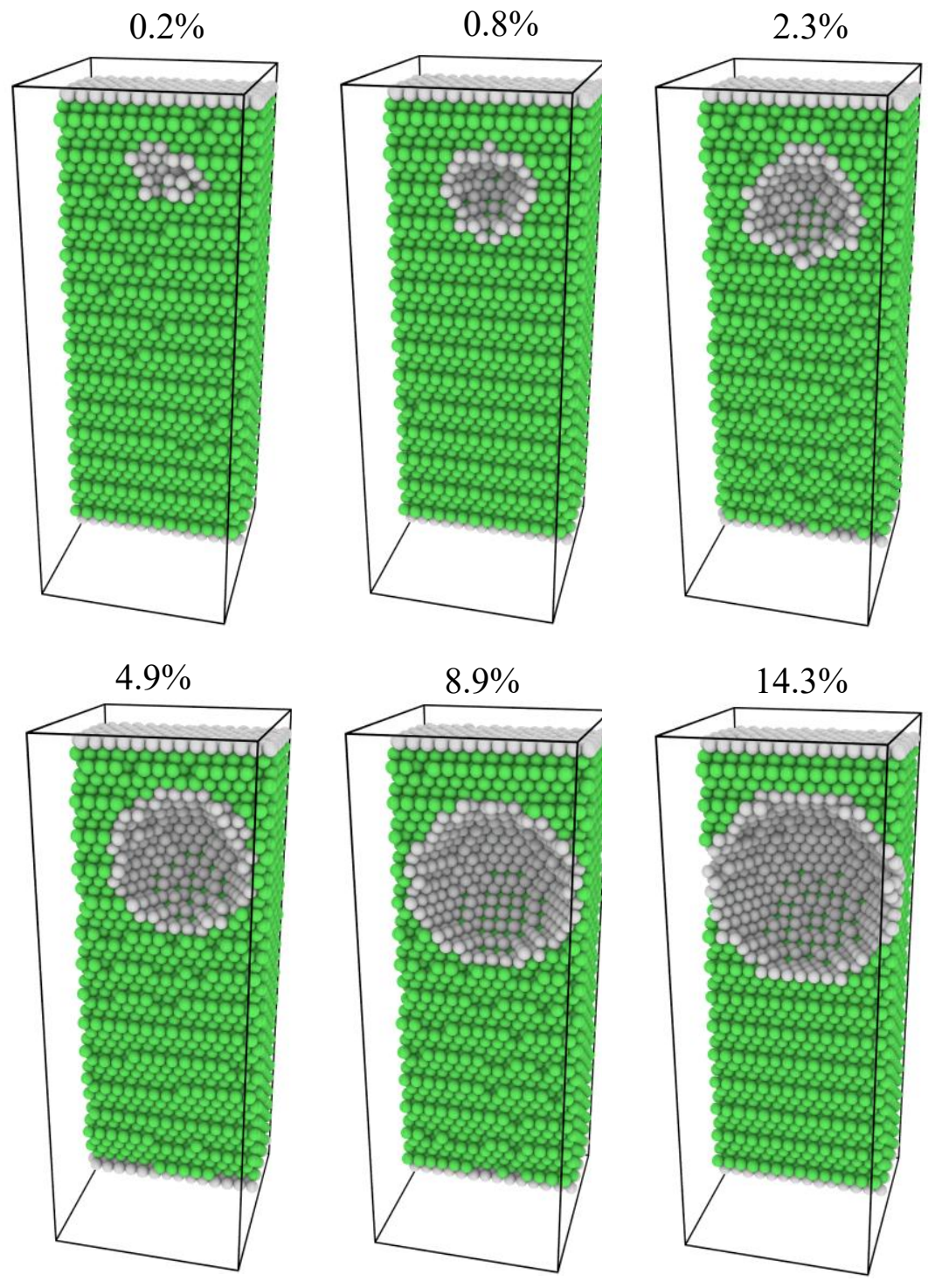

Figure S3. Simulated structures with increasing number fraction of clustered vacancies. Cutaways of the film structures are shown. Particles are colored by local environment (green=FCC and white=disordered). 
$0.1 \%$

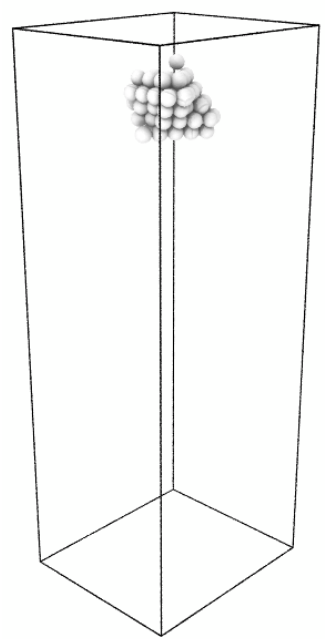

$0.3 \%$

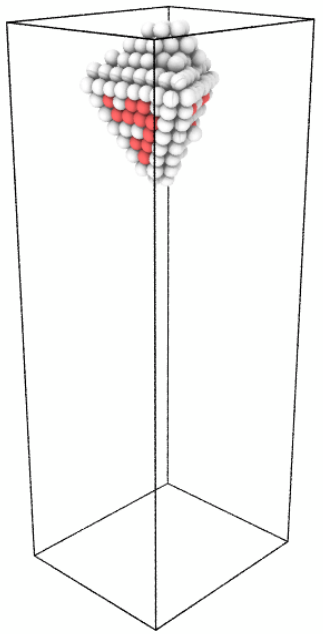

$0.6 \%$

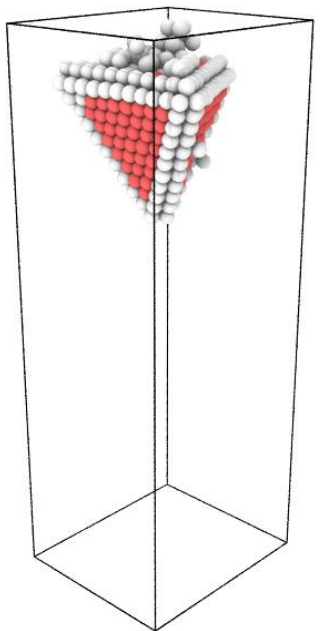

$1.0 \%$

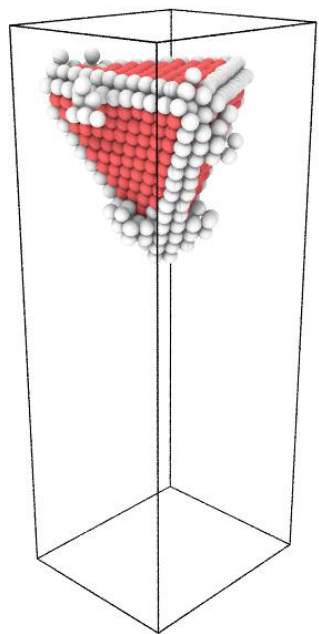

Figure S4. Simulated structures of stacking fault tetrahedra (SFT), produced by deleting increasing number fraction of particles. 

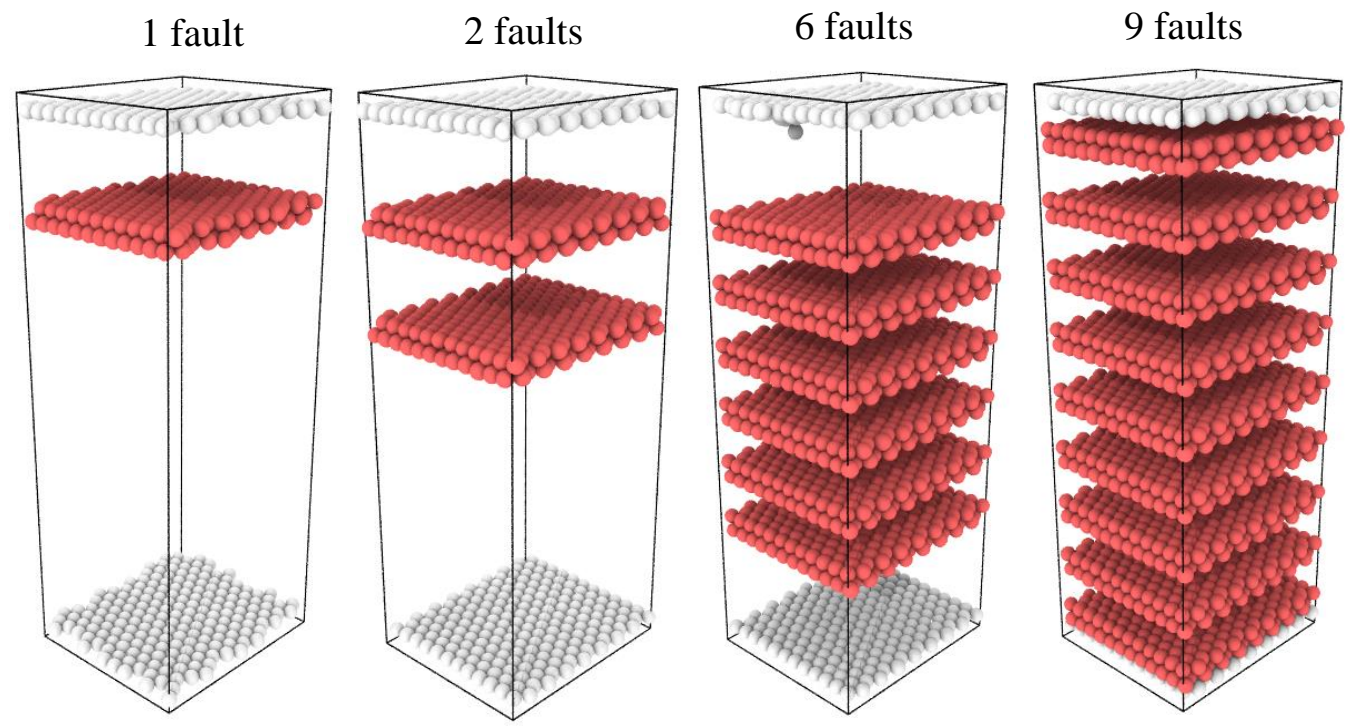

Figure S5. Simulated structures of planar stacking faults with increasing number of faults. 

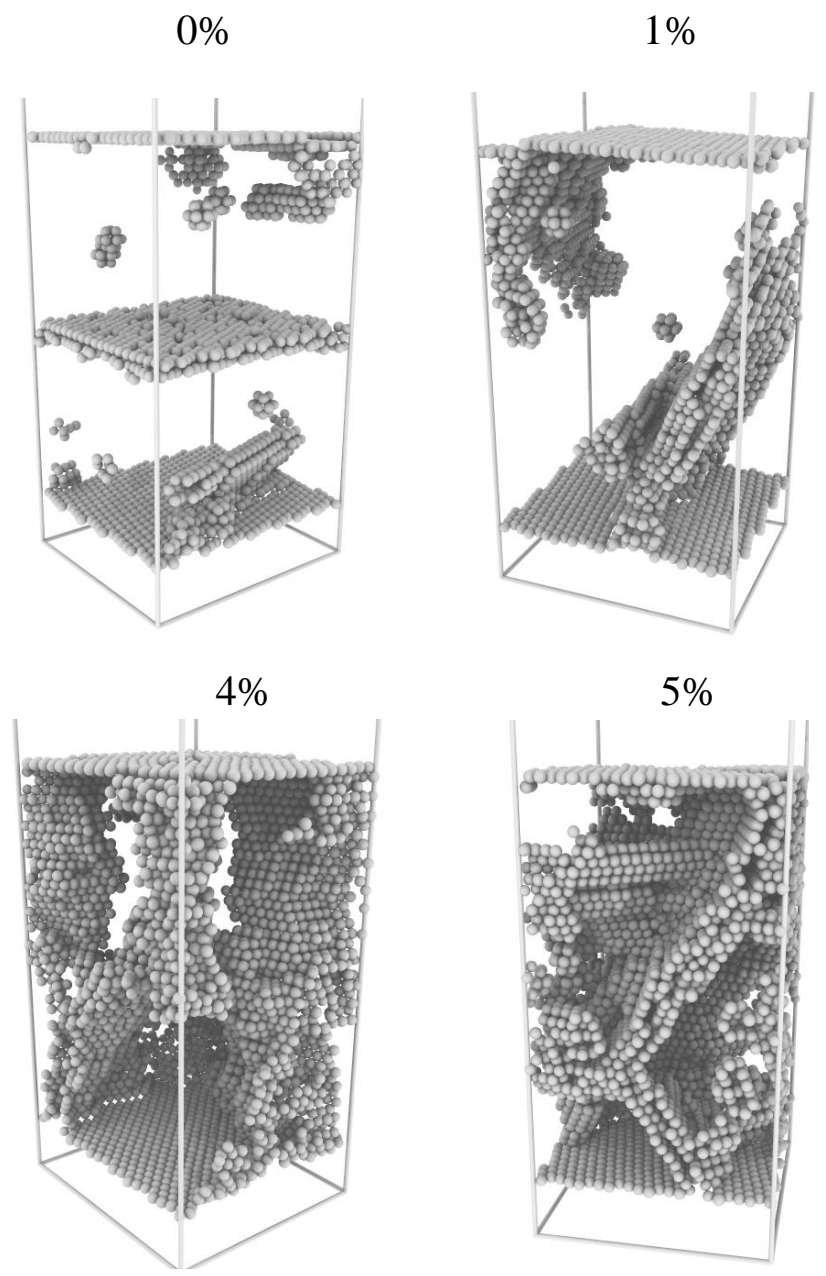

$2 \%$

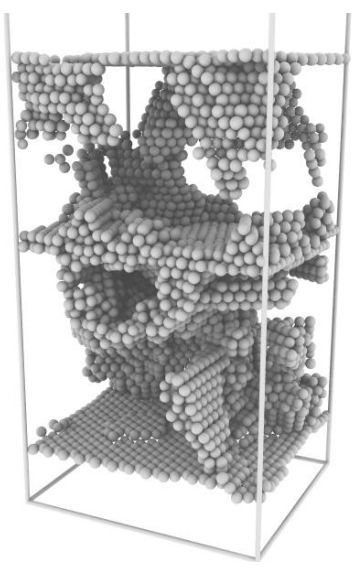

$7.5 \%$
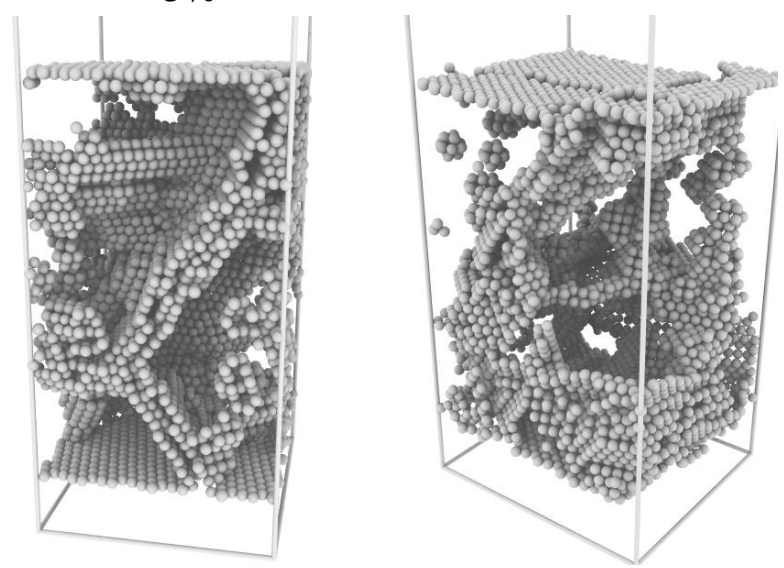

$3 \%$

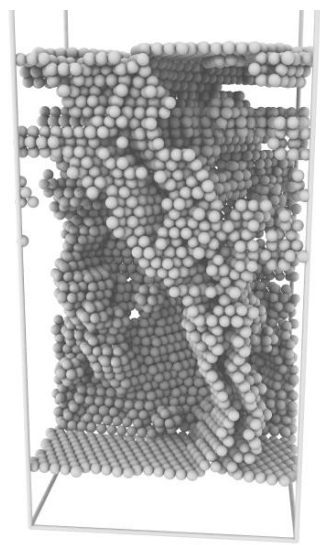

$10 \%$

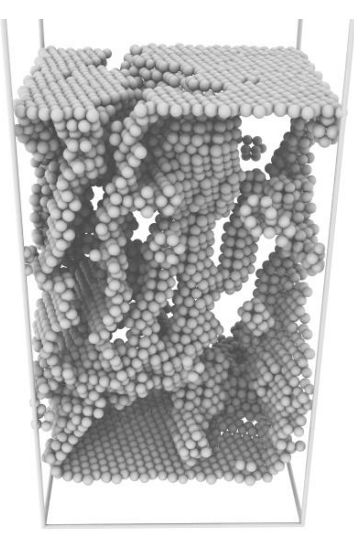

Figure S6. Simulated structures subjected to different amounts of biaxial strain, leading to microcracks. 